Gut, 1977, 18, 725-729

\title{
Gastric emptying of wholemeal and white bread
}

\author{
D.S. GRIMES ${ }^{1}$ AND J. GODDARD ${ }^{2}$ \\ From the Department of Medicine, Withington Hospital, Manchester, and the Department of Medical \\ Biophysics, University of Manchester, Manchester
}

SUMMARY We studied the rates at which solid and liquid leave the stomach after meals of wholemeal and white bread by using a double isotope technique. There was no difference in the rates at which the solid phases of the gastric contents left the stomach but liquid left the stomach significantly more rapidly with white bread than with wholemeal bread. Furthermore, the amount of liquid leaving the stomach unaccompanied and therefore unbuffered by solid was significantly greater after white bread than wholemeal bread. These findings may be of significance in the pathogenesis of duodenal ulcer and they provide a rational basis for a possible form of dietary treatment.

Carbohydrate refinement has been proposed to be of importance in the aetiology of duodenal ulcer (Cleave, 1962, 1974), but the suggestion that this might be due to loss of food buffer in the refining process has not been supported by experimental work (Tovey, 1974).

It has been suggested (George, 1968; Griffith et al., 1968)-although disputed (Cobb et al., 1971; Heading et al., 1976) - that patients with duodenal ulcer tend to have a more rapid rate of gastric emptying than normal but even if this phenomenon is real it is not known whether it is a cause or effect of the disease. We thought that the effect of carbohydrate refinement on gastric emptying in the normal subject should be investigated, as we thought it conceivable that herein might lie a possible link between carbohydrate refinement and duodenal ulcer. We expected that if this were so then gastric emptying would be more rapid after refined carbohydrate than after its unrefined counterpart.

Previous studies of the rates at which refined and unrefined carbohydrate leave the stomachs of normal subjects have produced conflicting results (McCance et al., 1953; Wilson et al., 1929) and, furthermore, they involved the measurement of the rate at which barium sulphate given with food left the stomach. By using radioactive tracer techniques which have recently become available (Griffith et al., 1966; Harvey et al., 1970) we have been able to

\footnotetext{
${ }^{1}$ Address for reprint requests: D. S. Grimes, Department of Medicine, Withington Hospital, Manchester M20 8LR.

${ }^{2}$ Present address: Department of Biophysics and Nuclear Medicine, Vanderbilt University, Nashville, Tennessee, USA.
}

Received for publication 16 February 1977 measure simultaneously the rates at which both the solid and the liquid components of a meal are emptied from the stomach.

\section{Method}

We studied 11 normal subjects, nine male and two female, aged between 20 and 30 years. All gave consent having been informed in detail of the nature of the investigation. The two females had been sterilised. Each ate meals of wholemeal and white bread in randomised sequence at intervals of one week. The meals were taken after an overnight fast.

The bread was prepared in the laboratory using a standard recipe of $120 \mathrm{~g}$ flour, $2 \mathrm{~g}$ sugar, $1 \mathrm{~g}$ salt, $1 \mathrm{~g}$ dried yeast, $1.5 \mathrm{ml}$ corn oil, $25 \mathrm{ml} \mathrm{milk}$, and $50 \mathrm{ml}$ water. Barbados sugar was used with wholemeal flour and white sugar with white flour. Approxi-

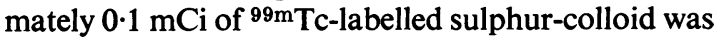
kneaded into each bread sample which was allowed to rise for one hour and then baked at $450^{\circ} \mathrm{C}$ for 30 minutes. The bread was allowed to cool and was then eaten lightly buttered and followed by a drink of $200 \mathrm{ml}$ water to which had been added approximately $0.1 \mathrm{mCi}$ of ${ }^{113 \mathrm{~m} I n}$-labelled DTPA (diethylene triamine penta-acetic acid).

For one hour after each meal the upper abdomen of the subject, in the recumbent position, was viewed by a gamma-camera linked to an on-line computer (MED II, Nuclear Data Inc.). Successive fiveminute images were accumulated for the peak energies of the gamma emission of both $113 \mathrm{~m}$ In and ${ }^{99 \mathrm{~m}} \mathrm{Tc}$ and saved on a magnetic disc. At the end of each study the gastric image was delineated on a 
screen with a light-pen and the total counts within this area on each of the 12 images were calculated by the computer. Empyting curves for both technetium and indium were produced by adjusting the initial counts for radioactive decay and the technetium curve was further corrected for indium crosstalk (that is the proportion of indium counts which are detected in the technetium channel). The emptying curves were subsequently normalised-that is, all points on a curve were expressed as a fraction of the maximum.

Eight rats were starved for $\mathbf{4 8}$ hours, fed bread labelled with $99 \mathrm{~m} \mathrm{Tc}$-sulphur-colloid, and killed one hour later. The stomachs were removed, opened and washed and the contents were centrifuged to separate solid and liquid. The relative counts of solid and liquid, the stomach itself, and the carcass minus the intestine were measured. In this way the efficacy of the technetium label was determined.

In vitro studies were performed to investigate the value of $113 \mathrm{~m}$ In-DTPA as a marker of the liquid phase. Samples of $6 \mathrm{~g}$ wholemeal and white bread were incubated at $37^{\circ} \mathrm{C}$ with $20 \mathrm{ml} 0.05 \mathrm{~N} \mathrm{HCL}$ and $0.02 \mathrm{mCi}{ }^{113 \mathrm{~m} I n-D T P A}$ for between five and 60 minutes. The mixture was then centrifuged at 6500 rpm for five minutes and the counts of the supernatant and the precipitate were measured. The weight of the initial solid was subtracted from the weight of the precipitate so as to determine the weight of the trapped or absorbed liquid. The counts of this liquid were estimated from the counts per gram of supernatant and subtracted from the counts of the precipitate so as to give the counts of the true solid. The uptake of ${ }^{113 \mathrm{~m} I n}$ by wholemeal and white bread was determined from the counts of the true solid expressed as a proportion of the total counts. This was called the fractional adsorption, $a$.

Seven subjects were studied to investigate the rate at which $200 \mathrm{ml}$ water labelled with $113 \mathrm{~m}$ In-DTPA left the stomach when taken alone without bread.

\section{Results}

As can be seen in Table 1, rat experiments demonstrated only a small loss of $99 \mathrm{~m} \mathrm{Tc}$ from bread and the differences between the losses from wholemeal and white bread are not statistically significant.

In vitro studies showed that the fraction of $113 \mathrm{~m}$ In adsorbed by wholemeal bread was $0.39 \pm$ SD 0.22 $(\mathrm{n}=80)$ and by white bread was $0.51 \pm$ SD 0.17 $(n=64)$. These values are significantly different at the $P<0.005$ level. They indicate that $113 \mathrm{~m}$ InDTPA is an imperfect marker of the liquid phase and to obtain a true liquid emptying curve we had to subtract from each point on the normalised indium curve the proportion of the solid phase which
Table 1 Loss of technetium from bread expressed as $\%$ of total Tc activity (mean $\pm S D, n=4$ )

\begin{tabular}{llll}
\hline & \multicolumn{3}{l}{ Loss to: } \\
\cline { 2 - 4 } & Liquid phase & Stomach wall & Carcass \\
\hline Wholemeal & $0.88 \pm 0.82$ & $3.50 \pm 3.61$ & $4.36 \pm 3.43$ \\
White & $1.44 \pm 1.24$ & $1.66 \pm 1.09$ & $0.60 \pm 0.84$ \\
Difference & NS & NS & NS \\
\hline
\end{tabular}

NS: not significant.

${ }^{113 m}$ In-DTPA would be labelling. The normalised indium curves were corrected by the equation:

$$
\operatorname{In} *(t)=\operatorname{In}(t)-a \cdot \operatorname{Tc}(t)
$$

where $\operatorname{In} *(t), \operatorname{In}(t)$ and $\operatorname{Tc}(t)$ represent the normalised

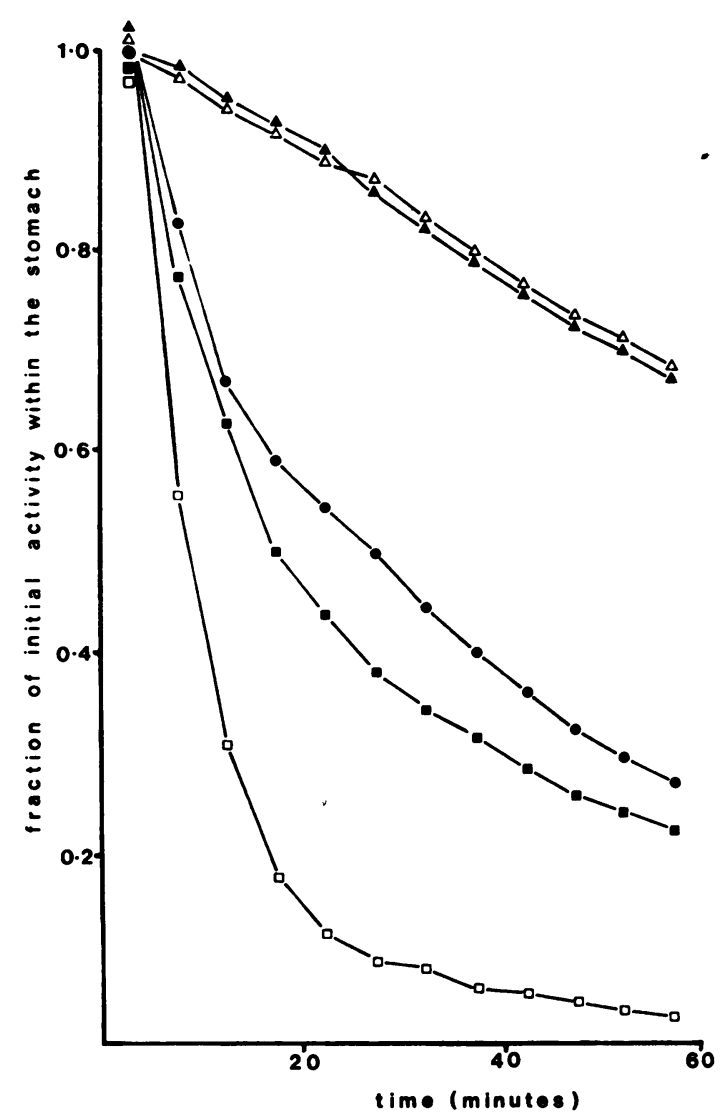

Figure Gastric emptying expressed as the fraction of the initial counts remaining over the gastric area at successive five-minute intervals. $\Delta$ Solid phase (wholemeal bread). $\triangle$ Solid phase (white bread). - Liquid phase (water alone). Liquid phase (wholemeal bread). $\square$ Liquid phase (white bread). Each point represents the mean of individual values in 11 subjects. 
Table 2 Rates of gastric emptying of solid and liquid expressed as emptying indices (see text for explanation)

\begin{tabular}{lll}
\hline Test meal & \multicolumn{2}{l}{ Emptying index $($ mean $\pm S D)$} \\
\cline { 2 - 3 } & Solid & Liquid \\
\hline $\begin{array}{l}\text { Wholemeal bread } \\
\mathbf{n}=11\end{array}$ & $0.032 \pm 0.022$ & $0.212 \pm 0.152$ \\
$\begin{array}{c}\text { White bread } \\
\mathbf{n}=11\end{array}$ & $0.032 \pm 0.01$ & $0.425 \pm 0.148$ \\
$\begin{array}{c}\text { Water alone } \\
\mathrm{n}=7\end{array}$ & & $0.150 \pm 0.130$ \\
\hline
\end{tabular}

values of the corrected and uncorrected indium curves and the technetium curves respectively at time $t$ and $a$ is the fractional adsorption of ${ }^{113} \mathrm{In}$.

These studies also demonstrated that the weight of water absorbed per gram of bread was $1.36 \pm$ SD $0.41 \mathrm{~g}(\mathrm{n}=80)$ for wholemeal bread and $1.49 \pm$ SD $0.21 \mathrm{~g}(\mathrm{n}=64)$ for white bread. The difference, although small, is significant at the $P<0.002$ level.

The points on the corrected emptying curves shown in the Figure have been analysed by the Wilcoxon matched-pairs signed-ranks test (twotailed) and probability level of $<0.05$ has been taken to indicate a significant difference (the first points on the curves were not analysed as they had all been normalised to one). There were no significant differences between any of the pairs of values of the points on the emptying curves of the solid phases of the wholemeal and white bread test meals. However, all pairs of values of the liquid phase emptying curves, were significantly different.

We have introduced the 'emptying index' to describe each curve. This is calculated from the formula $(1-f(T)) / A$ where $f(T)$ is the fraction of the initial volume remaining in the stomach at the end of the study at time $\mathbf{T}$ and $\mathrm{A}$ is the area under the emptying curve (an explanation is given below). The emptying indices have been calculated for each individual curve and again analysed by the Wilcoxon matchedpairs signed-ranks test. The mean values are shown in Table 2. The emptying indices for the solid phases of the wholemeal and white bread test meals are not significantly different. However, the emptying indices for the liquid phases of the wholemeal and white bread test meals are significantly different $(\mathrm{P}<$ $0.005, \mathrm{n}=11$ )

The points on the emptying curve of water taken alone have been compared with the corresponding points on the liquid phase emptying curves and analysed by the Mann-Whitney U test. There was no significant difference from the corresponding points of the liquid phase of the wholemeal bread test meal. Similarly, there was no difference in the emptying indices. However, all points were significantly different from corresponding points on the curve display- ing the emptying of the liquid phase of the white bread test meal $\left(\mathrm{P}<0.02, \mathrm{n}_{1}=6, \mathrm{n}_{2}=11\right)$ and the emptying indices were also significantly different $\left(\mathrm{P}<0.02, \mathrm{n}_{1}=6, \mathrm{n}_{2}=11\right)$.

\section{Discussion}

Whereas the results of McCance and colleagues (McCance et al., 1953) indicated that wholemeal bread left the stomach more rapidly than white bread our results show no difference. However, McCance took as his end point the time of complete emptying of the stomach whereas we studied the pattern of emptying during the first hour. McCance and colleagues observed that the barium image of the stomach after white bread was smaller and more concentrated than after wholemeal bread and this suggests a more rapid emptying of liquid after white bread, thus agreeing with the results of the present study.

The studies of Wilson and colleagues (Wilson et al., 1929) suggested that the more refined the food, the more rapidly it left the stomach but they did not use wholemeal bread as a test meal and it is perhaps unwise to extrapolate from one carbohydrate food to another. However, the problem arises as to what the barium was labelling. If it labelled the liquid phase to a significant degree then the results tend to conform with those of the present study.

It has previously been demonstrated that the greater the energy density of a meal the more rapid is the rate of gastric emptying (Hunt and Stubbs, 1975). The energy content of white bread is greater than that of wholemeal bread (3.49 and 3.33 Cals/g respectively, McCance and Widdowson, 1960) and therefore our results conform with this observation.

It is clear that in analysing the results of gastric emptying studies it is essential to know precisely what the labels are labelling. In vitro studies of label behaviour must be performed so that the results of the emptying studies have a meaning (Sheiner, 1975). The behaviour of the barium sulphate labels in the studies referred to above were not reported.

A further problem has been the expression of results (Sheiner, 1975). A single value expressing the rate of gastric emptying would be ideal. The halfemptying time is unsatisfactory, as it may necessitate the approximation of points to a curve and the extrapolation of that curve beyond the period of study. The expression of remaining volume at a given time loses much of the available data. The method of principal component analysis (Barber et al., 1975) may necessitate the approximation of points; it requires a computer for its calculation and it is not easily conceived by the non-mathematical mind. 
It is clear that the more rapidly the stomach empties the smaller is the remaining volume and quantity of label at successive intervals, and consequently the smaller will be the area under the curve representing that emptying (see Figure). There is thus an inverse relationship between the amount of emptying per unit time and the area under the curve. This can be demonstrated mathematically as follows:

Consider the stomach to contain a volume $\mathrm{V}$ and $\mathrm{a}$ quantity of label q, both leaving the stomach at rate $F$ which varies with time $t$.

$$
\therefore \frac{\mathrm{dq}}{\mathrm{dt}}=\mathrm{F}(\mathrm{t}) \cdot \mathrm{C}
$$

$$
\begin{array}{r}
\text { where } C=\frac{q(t)}{v(t)} \text { the concentration of the label } \\
\text { (assumed constant) }
\end{array}
$$

$$
\therefore \mathrm{dq}=\frac{\mathrm{F}(\mathrm{t}) .}{\mathrm{V}(\mathrm{t})} \mathrm{q}(\mathrm{t}) \cdot \mathrm{dt}
$$

assuming, as in many biological systems, that $\frac{F}{V}$ is constant and is a useful measure of motility

$$
\text { then } \begin{aligned}
\int_{0}^{T} d q=\frac{F}{V} \int_{0}^{T} q(t) . d t . \\
\therefore \frac{F}{V}=\frac{q_{0}-q_{T}}{\int_{0}^{T} q(t) d t}
\end{aligned}
$$

let $\quad f(t)=\frac{q(t)}{q_{0}}$ the fraction of label remain-

from $2 \quad \frac{F}{V}=\frac{1-f(T)}{A(T)}$

This we have called the 'emptying index': it represents the mean fractional change of volume or quantity in unit time. $f(T)$ is the fraction of label remaining in the stomach at the end of the emptying study and $\mathrm{A}(\mathrm{T})$ is the area under the curve representing the emptying.

The meaning of the emptying index can be further illustrated by the following derivation:

$$
\text { Mean emptying/unit time }=\frac{1}{N} \sum_{N}\left(q_{n}-q_{n+1}\right)
$$

where $\mathbf{N}$ is the number of observations and $\mathrm{q}_{\mathbf{n}}$ are individual observations of tracer in the stomach

$$
\begin{aligned}
& =\frac{1}{N}\left(q_{0}-q_{n}\right) \\
\text { Mean quantity of label } & =\frac{1}{N} \sum_{N} q_{n}
\end{aligned}
$$

$$
\begin{aligned}
\text { Mean emptying fraction/ } & =\frac{\mathrm{q}_{0}-\mathrm{q}_{\mathrm{n}}}{\sum_{N} \mathrm{q}_{\mathrm{n}}} \\
& =\frac{1-\mathrm{f}_{\mathrm{N}}}{\mathrm{A}_{\mathrm{N}}}
\end{aligned}
$$

For a normalised curve the theoretical maximum value for the emptying index is 1 , this implying a complete emptying of the stomach by the time of the second observation so that $f(T)=0$ and $A(T)=1$. The theoretical minimum value is 0 , this implying no emptying of the stomach by the end of the study so that $f(T)=1$ and $A(T)=N$, the number of observations.

The emptying index is thus a single value between 0 and 1 which is a measure of the motility of the stomach. It uses all the data available from the study and, unlike the rate of emptying, it is likely to be constant throughout the study. It does not involve extrapolation and it is independent of both the pattern of emptying and the distribution of points around the 'curve'. Moreover it is simple to calculate and easily understood.

A recent study of gastric emptying using two isotopes (Heading et al., 1976) indicated that during the period of the study the solid phase of the gastric contents left the stomach in a linear pattern, whereas the liquid phase left exponentially and more rapidly than the solid. This agrees with the results of the present study.

Our results demonstrate that liquid leaves the stomach more rapidly with white bread than with wholemeal bread. This suggests that a greater load of acid may enter the duodenum within the first $\mathbf{3 0}$ minutes of a meal of white bread and that the liquid leaving the stomach after this will have a higher hydrogen ion concentration. (Unfortunately it was not possible to monitor the intraduodenal $\mathrm{pH}$ and hydrogen ion content in this study.) These factors may be of importance in the pathogenesis of duodenal ulceration (George, 1968) supporting the concept that carbohydrate refinement might be a major cause of this condition (Cleave, 1962, 1974) and suggesting that the substitution of wholemeal bread for white bread might be of benefit to patients with duodenal ulcer and non-ulcer dyspepsia.

The question most obviously arising from our results is what is the mechanism of the difference in liquid phase emptying? Our observation that water taken with wholemeal bread leaves the stomach at a similar rate to water taken alone, whereas water taken with white bread leaves more rapidly, suggests that white bread actively accelerates liquid emptying, presumably by stimulating gastric motility to a degree which allows a more rapid passage of liquid across the pylorus but not solid. It might be 
expected that the fibre component of wholemeal bread would exert a drag effect on liquid emptying but our results do not support this. Moreover, we found that wholemeal bread did not absorb more water than white bread but we recognise the diffculties of measuring true water absorption and that test-tube phenomena may not be completely relevant to what happens within the stomach.

We would like to thank all the volunteers who made this study possible and Miss Anne-Marie McNeilly for her technical assistance. We would also like to thank Professor B. R. Pullen for his encouragement and advice, especially with the mathematics. Finally we would like to thank the Management Team of the Manchester AHA(T) South District who kindly provided a grant towards the study.

\section{References}

Barber, D. C., Duthie, H. L., and Howlett, P. J. (1975) Principal components: a new approach to the analysis of gastric emptying. In Dynamic Studies with Radioisotopes in Medicine, vol. 1, pp. 185-195. International Atomic Agency: Vienna.

Cleave, T. L. (1962). Peptic Ulcer. Wright: Bristol.

Cleave, T. L. (1974). The Saccharine Disease. Wright: Bristol.

Cobb, J. S., Bank, S., Marks, I. N., and Loun, J. H. (1971). Gastric emptying after vagotomy and pyloroplasty. American Jcurnal of Digestive Diseases, 16, 207-215.
George, J. D. (1968). Gastric acidity and motility. American Journal of Digestive Discases, 13, 376-383.

Griffith, G. H., Owen, G. M., Kirkman, S., and Shields, R. (1966). Measurement of rate of gastric emptying using chromium-51. Lancet, 1, 1244-1245.

Griffith, G. H., Owen, G. M., Campbell, H., and Shields, R. (1968). Gastric emptying in health and gastroduodenal disease. Gastroenterology, 54, 1-7.

Harvey, R. F., Mackie, D. B., Brown, N. J. G., Keeling, D. H., and Davies, W. T. (1970). Measurement of gastric emptying time with a gamma camera. Lancet, 1, 16-18.

Heading, R. C., Tothill, P., McLoughlin, G. P., and Shearman, D. J. C. (1976). Gastric emptying rate measurement in man. A double isotope scanning technique for simultaneous study of liquid and solid components of a meal. Gastroenterology, 71, 45-50.

Hunt, J. N., and Stubbs, D. F. (1975). The volume and energy content of meals as determinants of gastric emptying. Journal of Physiology, 245, 209-225.

McCance, R. A., Prior, K. M., and Widdowson, E. M. (1953). $A$ radiological study of the rate of passage of brown and white bread through the digestive tract of man. British Journal of Nutrition, 7, 98-104.

McCance, R. A., and Widdowson, E. M. (1960). The Composition of Foods, p. 25. M.R.C. Special Report Series No. 297. HMSO: London

Sheiner, H. J. (1975). Progreșs report: gastric emptying tests in man. Gut, 16, 235-247.

Tovey, F. I. (1974). Aetiology of duodenal ulcer: an investigation into the buffering action and effect of pepsin on bran and unrefined carbohydrate foods. Postgraduate Medical Journal, 50, 683-688.

Wilson, M. J., Dickson, W. H., and Singleton, A. C. (1929). Rate of evacuation of various foods from the normal stomach. Archives of Internal Medicine, 44, 787-796. 